\title{
Diffusion and Aggregation of Sodium Fluorescein in Aqueous Solutions
}

\author{
Tommaso Casalini, Matteo Salvalaglio, Giuseppe Perale, Maurizio Masi, and Carlo Cavallotti* \\ Dipartimento di Chimica, Materiali e Ingegneria Chimica “G. Natta”, Politecnico di Milano via Mancinelli 7-20131 Milano, Italy \\ Supporting Information
}

ABSTRACT: The diffusion and aggregation of sodium fluorescein in aqueous solutions was investigated adopting density functional theory (DFT) and molecular dynamics (MD) simulations. First, DFT calculations in implicit water were used to determine minimum energy structure and atomic charges of the solute, which were then used as input for explicit water MD simulations. The self-diffusion coefficient of sodium fluorescein was calculated using the Einstein equation, computing the mean square displacement from $24 \mathrm{~ns}$ trajectories. The calculated diffusion coefficient, $0.42 \cdot 10^{-5} \mathrm{~cm}^{2} \mathrm{~s}^{-1}$, is in good agreement with literature experimental data. The simulations confirmed the tendency of fluorescein to form dimers. In order to achieve a

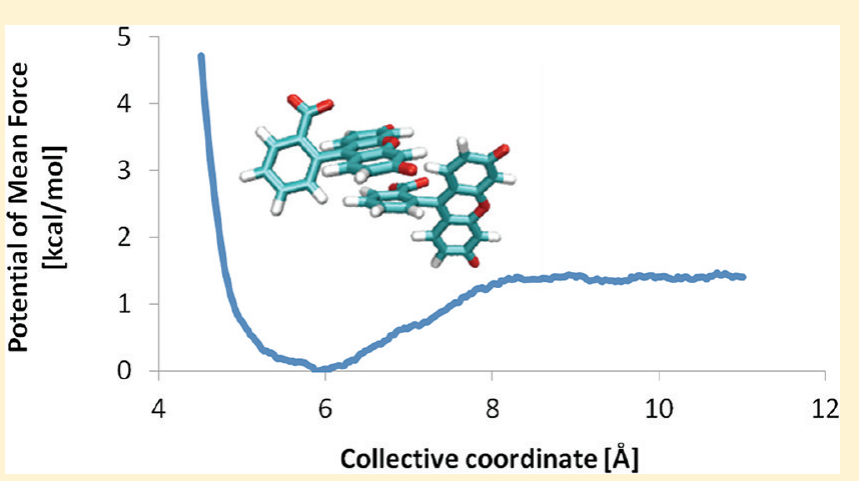
deeper understanding of aggregation phenomena, the dimer geometry was investigated through DFT calculations both in vacuo and in implicit water using different functionals and solvation theories. The results showed that dimerization does not occur in vacuo, as charge repulsion dominates, and that the minimum energy dimer structure is symmetric and stabilized by edge-to-face $\pi-\pi$ interactions. The interaction energy was computed both at the DFT level and through MD simulations using Umbrella Sampling. The free interaction energy calculated with the WHAM and Umbrella Integration protocol, $-1.3 \mathrm{kcal} / \mathrm{mol}$, is in good agreement with experimental data, while the value determined using DFT calculations is significantly smaller and depends largely from the chosen functional and the computational methodology used to determine the solute-solvent boundary surface.

\section{INTRODUCTION}

The importance of fluorescein for chemical analysis is determined by its fluorescence, which makes it useful for many applications as it is easily detectable with UV spectroscopy. Among the several possible applications, it is used in ophthalmology, in order to detect corneal diseases, and as a drugmimetic compound, since it has a molecular weight and a steric hindrance similar to commonly used drugs. These features make sodium fluorescein a good candidate to investigate the release from biomedical devices. ${ }^{1,2}$ This is indeed the application of this molecule that sparkled the basis of the present investigation. Indeed, this compound was used in previous works to characterize the release behavior of molecules loaded in hydrogels. ${ }^{3}$ In particular, hydrogels made of Agarose (a polysaccharide) and Carbomer (a poly acrylic acid) were deeply investigated, seen their potentiality as drug delivery tools in regenerative medicine. ${ }^{4,5}$ A consistent experimental activity was then devoted to measure the diffusion coefficient of fluorescein in such gels and in water. ${ }^{2,3}$ One of the most intriguing results was that, counterintuitively, the sodium fluorescein diffusion measured in the gel is larger with respect to that it has in water. Moreover, the solute diffusivity increased with its concentration and the network mesh size. $^{2}$ It is from this observation that the present study takes its motivation. While the final aim is to explain at a molecular level the origin of the diffusion enhancement effect through molecular dynamics (MD) simulations, the present work is the first step, aimed at studying the molecular mechanism of diffusion of fluorescein in water as well its tendency to aggregate in dimers, which is known to occur significantly when its concentration reaches a critical level. ${ }^{6,7}$

Because of its importance, fluorescein molecular properties have been largely studied in the scientific literature. It is thus known that fluorescein possesses a complex acid-base equilibrium in aqueous solutions and can exist in four different forms: cationic, neutral, anionic, and dianionic. ${ }^{8}$ This equilibrium has a strong dependence on $\mathrm{pH}$. Sjöback et al. ${ }^{9}$ studied fluorescein protolytic equilibria with spectroscopic techniques; they found that at neutral and basic $\mathrm{pH}$ anionic and dianionic forms are present, while at acidic $\mathrm{pH}$, the neutral and cationic species are most abundant. Moreover, the acid-base equilibrium is coupled with a tautomeric equilibrium, since two different anionic forms and three different neutral forms are possible. Literature offers some examples of computational efforts to compute ionization and tautomerization constants. Kròl et al. ${ }^{10}$ calculated ionization

\footnotetext{
Received: August 4, 2011

Revised: September 28, 2011

Published: September 29, 2011
} 
and tautomerization constants at different levels of theory in implicit water, using the Conductor Polarizable Continuum Model (CPCM). Jang et al. ${ }^{11,12}$ used density functional theory (DFT) at the B3LYP $/ 6-311+G(d, p)$ level to determine the population of neutral and anionic tautomers in vacuo, in implicit water and in implicit DMSO with a Poisson-Boltzmann continuum solvation approach. Batistela et al. ${ }^{13}$ performed similar computations at the B3LYP/3-21+G(d) level of theory in implicit water. Their results, however, are not in full agreement for what concerns the predicted relative amount of the ions. In the present work, the focus is on the properties of the sodium salt of fluorescein. Sodium fluorescein possesses a high solubility in water, equal to $500 \mathrm{mg} \mathrm{mL}{ }^{-1}$, so that in solution it is fully dissociated in two sodium ions and a fluorescein molecule, which is in equilibrium between the anionic and dianionic state. At a neutral $\mathrm{pH}$, the concentration of the anion is significantly smaller than that of the dianion (between 10 and $15 \%$ for a $\mathrm{p} K_{\mathrm{a}}$ of $6.1-6.3$ ).

The fluorescein self-diffusion coefficient in water has been experimentally measured by several authors. Saltzman et al. ${ }^{14}$ determined a self-diffusion coefficient of $0.57 \pm 0.07 \times 10^{-5} \mathrm{~cm}^{2}$ $\mathrm{s}^{-1}$ through computer imaging of fluorescence profile, while Radomsky et al. ${ }^{15}$ reported a value of $0.51 \times 10^{-5} \mathrm{~cm}^{2} \mathrm{~s}^{-1}$. Soeller et al. ${ }^{16}$ using two-photon flash photolysis technique found a value of $0.4 \times 10^{-5} \mathrm{~cm}^{2} \mathrm{~s}^{-1}$. In our recent experimental activity, ${ }^{3}$ a sodium fluorescein self-diffusion coefficient of $0.39 \pm$ $0.04 \times 10^{-5} \mathrm{~cm}^{2} \mathrm{~s}^{-1}$ was determined through NMR spectra.

Spectroscopic data suggest that dianionic fluorescein is able to form aggregates ${ }^{17}$ even at low concentrations. Indeed the UV absorption spectra of fluorescein dianion changes by increasing its concentration because of aggregation phenomena. Starting from spectroscopic data, both Rohatgi and Singhal ${ }^{18}$ and Lòpez Arbeloa ${ }^{6,19,20}$ calculated the equilibrium constant for dianionic fluorescein dimerization and determined a Gibbs free energy of aggregation of about $-1 \mathrm{kcal} \mathrm{mol}^{-1}$. The data were taken in similar conditions. Both measurements were performed in water at a $\mathrm{pH}$ of 12 and at fluorescein concentrations comprised between $10^{-2}$ and $5.0 \times 10^{-1} \mathrm{~mol} \mathrm{~L}^{-1}$. The only notable difference is that Lopez Arbeloa performed all of the measurements in $0.01 \mathrm{~mol} \mathrm{~L}^{-1}$ of KCl, while Rohatgi and Singhal do not clearly define the solution composition. Lòpez Arbeloa proposed also that trimeric forms ${ }^{21}$ can exist at high dianionic fluorescein concentrations (i.e., higher than $0.5 \mathrm{~mol} \mathrm{~L}^{-1}$ ), and computed a free energy of trimerization of $-1.4 \mathrm{kcal} / \mathrm{mol}$ from experimental spectroscopic data. ${ }^{7}$ Both Rohatgi ${ }^{22}$ and Lòpez Arbeloa ${ }^{6}$ proposed possible dimer structures in terms of distances between the monomer centers of mass and angles between its dipole moments on the basis UV absorption spectra analyzed through exciton interaction theory. ${ }^{22} \mathrm{De}$ et $\mathrm{al}^{23}$ characterized dimerization phenomena by means of UV absorption spectra in ethanol and DMSO, finding that protic solvents promote aggregation. The literature is not rich of computational studies of dianionic fluorescein dimers, as the focus of computational investigations was mostly on other xanthene dyes. Das et al. ${ }^{24}$ studied fluorescein dimerization in bile salt hydrogels, proposing a geometry obtained through AM1 calculations. Daré-Doyen et al. ${ }^{25}$ analyzed the dimerization in water of two xathene dyes, Rhodamine 6G (R6G) and Pyronine 6G, through molecular dynamics simulations using the AMBER ff94 force field. They were able to find dimer structures and to characterize them in terms of geometry and interaction energy. A similar analysis was carried out by Chuichay et al. ${ }^{26}$ The dimerization free energy calculated
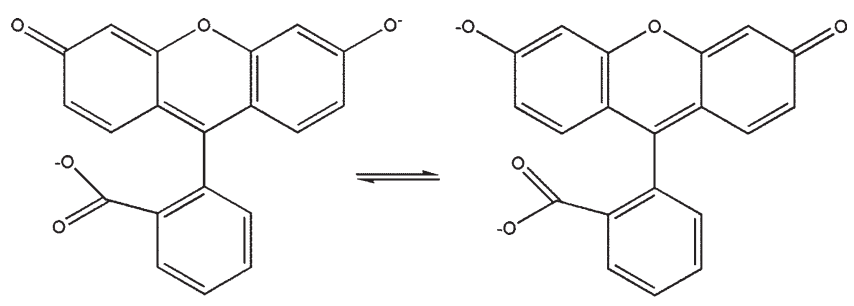

Figure 1. Resonant atoms of dianionic fluorescein.

through thermodynamic integration for the R6G dimer was $-7 \mathrm{kcal} / \mathrm{mol}$. The computational analysis was successively extended by the same authors to study the interaction of R6G with DNA ${ }^{27}$ R6G aggregation was confirmed through NMR experimental data by Ilich et al. ${ }^{28} \mathrm{~A}$ similar computational analysis is however not reported in the literature for sodium fluorescein.

As said, the aim of this work is to study the dynamic behavior of sodium fluorescein in solution. In the first part of this study, the analysis is focused on the computation of the self-diffusion coefficient. The second part is aimed at studying aggregation phenomena both through DFT and MD computations.

\section{METHODS AND THEORETICAL BACKGROUND}

The investigation of sodium fluorescein dynamics was performed using different computational approaches. First, the structure of dianionic fluorescein was obtained by means of quantum chemistry adopting DFT calculations, successively diffusion and dimerization phenomena were analyzed using both $\mathrm{MD}$ and DFT simulations.

Fluorescein Structure and Force Field. The structure of dianionic fluorescein was determined at the DFT level using the Becke three parameters ${ }^{29}$ and Lee-Yang-Parr functionals ${ }^{30}$ to calculate exchange and correlation energies. The calculations were performed using the Gaussian $09^{31}$ suite of program. First, the structure was optimized in vacuo at the B3LYP $/ 6-31 \mathrm{G}(\mathrm{d}, \mathrm{p})^{32}$ level. The so determined geometry was then used as starting point for optimization in water at the same level of theory. Water was modeled as an implicit solvent using the integral equation formalism polarizable continuum model (IEF-PCM) ${ }^{33}$ at a temperature of $300 \mathrm{~K}$. Structures and molecular properties so determined were used to build a force field, compatible with the Amber ff03 force field ${ }^{34}$ to be used in the MD simulations of fluorescein. Atomic charges were then determined from electrostatic potentials (ESPs), calculated at the B3LYP $/ 6-311+G(\mathrm{~d}, \mathrm{p})^{35}$ level of theory in implicit water. The charges were fitted using the RESP ${ }^{36,37}$ formalism in two steps. First, a charge of -2 was assigned to dianionic fluorescein. Then, charge equivalence for chemically equivalent atoms was imposed. In order to assign correct atomic charges, it was considered that the molecule has four resonance structures, so that the two negative charges are spread over four $\mathrm{O}$ atoms, as shown in Figure 1.

Finally, atomic types, connectivities, interatomic distances, and angles were collected in a library and assigned on the basis of atomic types defined in the $\mathrm{ff} 03$ force field. Structures and libraries of fluorescein are reported as Supporting Information to this paper.

Molecular Dynamics Simulations. Molecular dynamics simulations were performed adopting the $\mathrm{ffO} 3$ force field and Amber $10 .^{38}$ Simulations were performed in the NPT ensemble considering 30 molecules of dianionic fluorescein contemporarily that were solvated using about 14000 explicit TIP3P ${ }^{39}$ water 
molecules in a solvent box with an initial edge of $80 \AA$. A dielectric constant equal to 1 was used for all of the simulations performed in explicit water and the cutoff distance for long-range interaction was set to $15 \AA$. In order to impose electroneutrality, and to take into account the presence of counterions, $60 \mathrm{Na}^{+}$ions were added. All simulations were performed with periodic boundary conditions and long-range interactions were computed with the Particle Mesh Ewald (PME) method. The computational protocol adopted for MD simulations was the following: first, a 2000-cycle energy minimization was carried out in order to remove bad contacts between the solute and the random-placed solvent molecules. In this step, solute molecules were restrained with a harmonic potential $k(\Delta x)^{2}$ with $\mathrm{k}$ set to $500 \mathrm{kcal} \mathrm{mol}^{-1} \AA^{-2}$. A second 3500-cycle energy minimization was performed for the entire system, removing the previous restraints. Then, the temperature was raised from 0 to $300 \mathrm{~K}$ by a simulated annealing of $20 \mathrm{ps}$ at constant volume; a weak harmonic restraint (where $\mathrm{k}$ is now equal to $10 \mathrm{kcal} \mathrm{mol}^{-1} \AA^{-2}$ ) was applied on the solute with the purpose of avoiding wild fluctuations. This was followed by a 100 ps run at constant pressure, in order to allow the system relaxation and thus to reach the correct density. Finally, MD simulations of 24 ns were performed at $300 \mathrm{~K}$ and atmospheric pressure. Temperature and pressure control was performed through Langevin dynamics with a collision frequency of $1 \mathrm{ps}^{-1}$ and isotropic position scaling, respectively. The SHAKE ${ }^{40}$ algorithm was used for covalent bonds involving hydrogen atoms, allowing to adopt a time step of 2 fs. Molecules coordinates were collected every 4 ps. The diffusion coefficient was computed by means of Einstein equation: ${ }^{41}$

$$
D=\lim _{t \rightarrow \infty} \frac{1}{6 t}\left\langle\left[(r(0)-r(t)]^{2}\right\rangle\right.
$$

Where $D$ is the diffusion coefficient and the term into brackets is the mean square displacement (MSD) of the molecule, which was averaged over all the 30 solute molecules. The limit operator, where time tends to infinite, indicates that this relation is valid for long time scales, when the diffusion regime has been reached. The attainment of the diffusion regime has been checked by increasing the simulation time up to the point at which the angular coefficient of $\log (t)$ vs $\log (\mathrm{MSD})$ is equal or close to one.

Fluorescein Dimer Structure. The minimum energy structure of the fluorescein dimer was determined using DFT adopting the M06-2 $\mathrm{X}^{42,43}$ density functional, which accounts for dispersion interactions. ${ }^{43,44}$ Computations were performed using the $6-311+G(2 d, 2 p)^{45}$ basis set both in vacuo and in water. Water was modeled as an implicit solvent using the IEF-PCM model at $300 \mathrm{~K}$. The molecular surface representing the solute-solvent boundary was described both using Solvent Accessible Surface (SAS) and the van der Waals (VdW) surfaces. ${ }^{46}$ Once the minimum energy geometry had been obtained, it was used to perform single point energy calculations at different levels of theory.

Dimerization Potential of Mean Force. The free energy profiles associated with the formation of sodium fluorescein dimers at $\mathrm{pH} 7$ and 12 were computed employing an Umbrella Sampling (US) strategy. ${ }^{47-49}$ The US simulations were performed applying periodic boundary conditions in a box of $50 \times$ $50 \times 50 \AA$ comprising approximately 4500 TIP3P water molecules. A $10 \AA$ cutoff was employed and long-range electrostatic interactions were computed with the Particle Mesh Ewald approach. Simulations were performed at constant temperature and pressure, kept at $300 \mathrm{~K}$, and $1 \mathrm{bar}$, respectively, employing
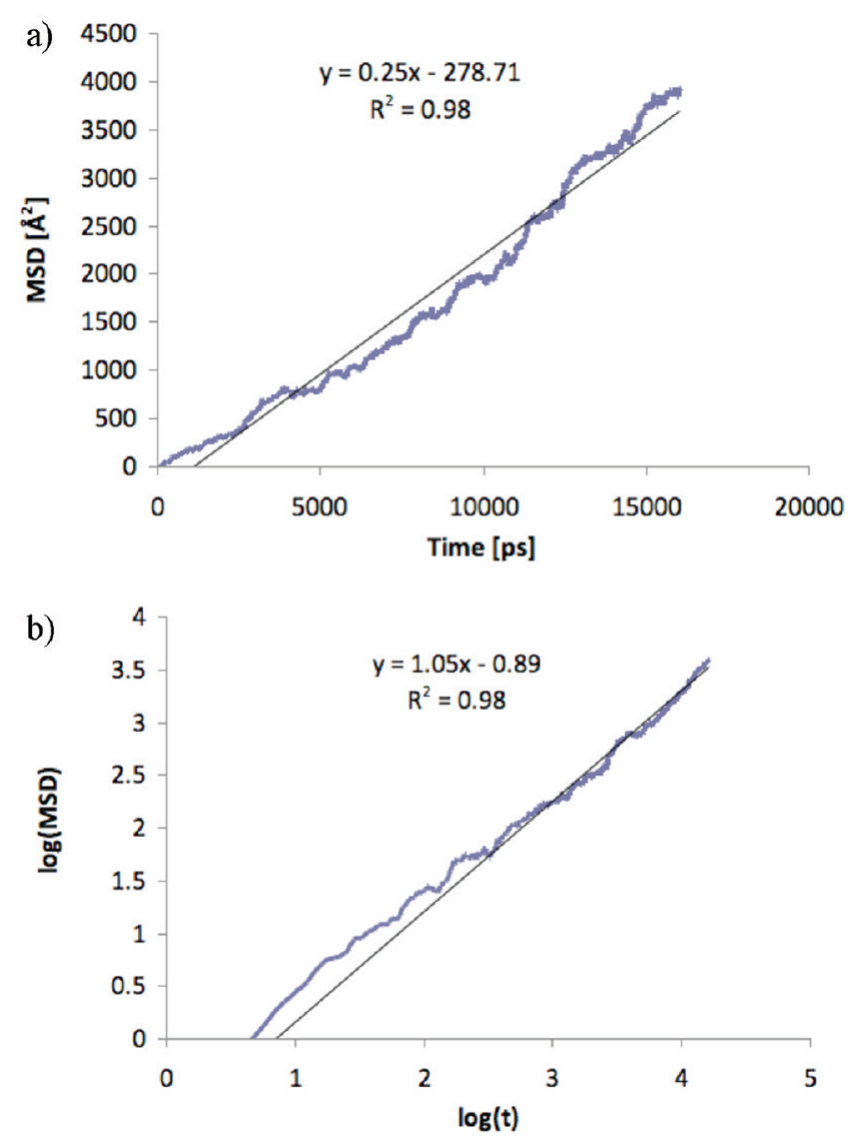

Figure 2. Dependence of mean square displacement from simulation time for sodium fluorescein (a) and the respective logarithmic plot (b).

Langevin dynamics with a collision frequency of $1 \mathrm{ps}^{-1}$ and isotropic position scaling. The $\mathrm{SHAKE}^{40}$ algorithm was applied to restrain the bonds involving hydrogen, thus allowing for an integration step of $2.0 \mathrm{fs}$. Each production run was extended for $2 \mathrm{~ns}$ and was preceded by a 2000 cycles minimization. MD simulations have been performed using the ff03 Amber force field $^{34}$ as implemented in the Amber 10 computational suite. ${ }^{38}$ Harmonic biasing potentials $k(\Delta x)^{2}$ with a $k$ value of $15 \mathrm{kcal}$ $\mathrm{mol}^{-1} \AA^{-2}$ were applied to enhance the sampling along a collective coordinate representative of the association/dissociation pathway. Such a collective coordinate was straightforwardly defined as the distance between the centers of mass of the fluorescein molecules mutually interacting in the dimer. The collective coordinate was sampled in the 4-11 $\AA$ range with an $0.5 \AA$ spacing between adjacent windows. Collective coordinate values were collected at each integration step and postprocessed via $\mathrm{WHAM}^{47,48}$ and Umbrella Integration algorithms. ${ }^{49}$ Systematic and statistical errors associated with the histogram-based reconstruction of the probability distribution along the collective coordinate were evaluated according to the approach proposed by Kobrak; ${ }^{50}$ the bin size was thus designed to maintain the intrinsic methodological error below the $0.1 \mathrm{kcal} / \mathrm{mol}$ threshold.

\section{RESULTS AND DISCUSSION}

Fluorescein Diffusion Coefficient. The diffusion coefficient was computed through the Einstein equation, using the mean square displacement calculated from all the molecular trajectories 

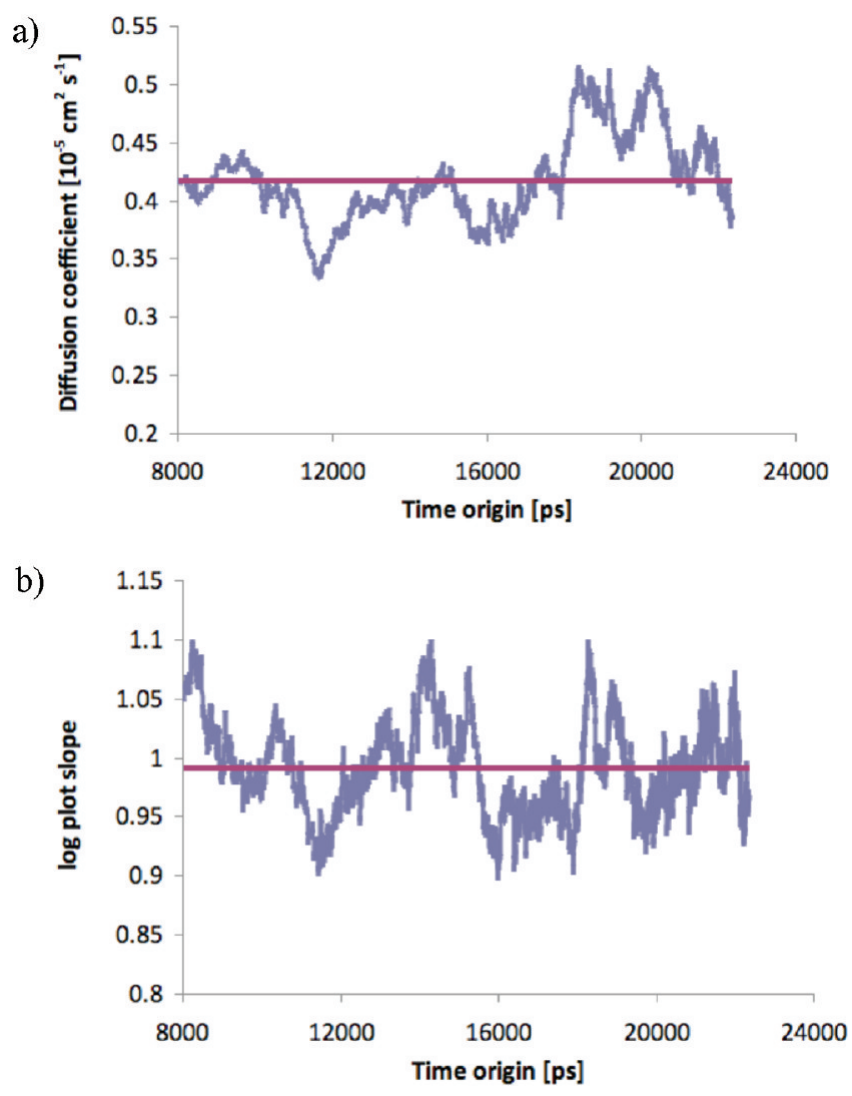

Figure 3. Diffusion coefficient fluctuations with respect to a change of the simulation time from which it is computed (a) and the respective $\log (t) / \log (\mathrm{MSD})$ plot.

collected during the simulations determined with respect to the center of mass of each considered molecule. The choice of the number of fluorescein molecules considered and of the simulation volume was instrumental, as it allowed to reproduce, after system equilibration, the sodium fluorescein concentration $\left(0.132 \mathrm{~mol} \mathrm{~L}^{-1}\right)$ that corresponds to an experimental condition in which the diffusion coefficient was recently measured. ${ }^{3}$ The plot of $\log$ (MSD) vs $\log (t)$ calculated as a function of the simulation time is shown in Figure 2. Analyzing the molecular trajectories, the diffusion regime was reached after $8 \mathrm{~ns}$. Indeed, taking this point as time origin to compute the mean square displacement, the slope of the $\log (t)$ vs $\log (\mathrm{MSD})$ plot is equal to 1.05 and the corresponding diffusion coefficient, determined through eq 1 , is $0.42 \times$ $10^{-5} \mathrm{~cm}^{2} \mathrm{~s}^{-1}$, which is in good agreement with the experimental value $^{3}$ of $0.39 \times 10^{-5} \mathrm{~cm}^{2} \mathrm{~s}^{-1}$.

The analysis of the simulations shows that after $8 \mathrm{~ns}$, independently from the time origin, the values of log plot slope and diffusion coefficient fluctuate with an average value of $0.99 \pm$ 0.04 and $0.42 \pm 0.04 \cdot 10^{-5} \mathrm{~cm}^{2} \mathrm{~s}^{-1}$, respectively, as shown in Figure 3. Fluctuations are due to statistical noise.

Several simulations were performed for comparable time spans to investigate the influence of the cut off value used for the calculation of intermolecular interactions. It was found that the diffusion coefficient has a slight but significant dependence from the cutoff, since it changes from $0.47 \times 10^{-5} \mathrm{~cm}^{2} \mathrm{~s}^{-1}$ to $0.53 \times 10^{-5} \mathrm{~cm}^{2} \mathrm{~s}^{-1}$ increasing the cut off from 8 to $10 \AA$, respectively.

The analysis of the trajectories showed that during the simulations dianionic fluorescein spontaneously forms aggregates, which can be attributed to the establishment of electrostatic and van der Waals interactions. In particular, not only the formation of dimers was observed in the simulated time span, but also that of a trimer. The presence of trimeric aggregates has been proposed in the literature on the basis of UV spectroscopical data, 6,7 but it is considered to be negligible at the low concentrations investigated in this work. Indeed, during the simulations only one trimeric aggregate was observed, with a short lifetime of about $800 \mathrm{ps}$. In order to evaluate whether the presence of dimers may affect the diffusion coefficient, its value was recalculated considering separately in the computation of the MSD the molecules that formed dimers and the others. As no significant deviation was observed, it can be thus concluded that the diffusion coefficient here reported is not affected by the formation of aggregates.

Analysis of the Sodium Fluorescein Dimer:DFT Calculations. The minimum energy structure of the fluorescein dimer was determined using DFT calculations at the M06-2X/6-311+G$(2 \mathrm{~d}, 2 \mathrm{p})$ level. Simulations were performed both in vacuo and in water. It was found that the use of a density functional able to account for dispersion interactions is fundamental, as this contribution is crucial for the formation of an energetically stable dimer. The fluorescein mutual intermolecular interaction can in fact be rationalized in terms of a competition between electrostatic forces, which are repulsive, and an attractive component due to van der Waals forces. In the gas phase electrostatic repulsion dominates, so that no energetically stable dimer structure could be found, independently from the functionals used to compute exchange and correlation energy. In contrast, DFT geometry optimizations performed in water converged to a dimer structure both when using functionals that do not account for dispersion forces, such as the B3LYP functionals, as well as when using functionals in which the dispersion contribution is considered, as is the case for the M06-2X functionals. However, the interaction energies were here found to significantly depend both from the chosen functional, as was expected, and from the methodology adopted to determine the molecular surface representing the solute-solvent boundary. The influence of the type of boundary molecular surface is significant, as it impacts both the structure and the energy of the dimer structure. As specified in the Methods Section, two different approaches were used. The first, defined by the keyword SAS in G09, creates the boundary surface by adding to the radius of each solute atom that of the solvent, whereas in the second, named VDW, the solvent radius is set to 0 . The structures of the dimers calculated using the two approaches are shown in Figures 4 and 5, while their $x y z$ coordinates are reported as Supporting Information. As it can be observed, both structures have a C2 symmetry axis and are characterized by the formation of different $\pi-\pi$ interactions involving the aromatic rings. The two structures differ significantly for what concerns the distance between the centers of mass, which goes from $6.64 \AA$ for the structure determined using the SAS surface to $6.16 \AA$ for that determined using the VDW surface.

Two different $\pi-\pi$ interactions are present in the dimer: a partial parallel $\pi-\pi$ stacking involving the two benzoic rings and two edge-to-face interactions between one benzoic ring and one of the two phenoxy rings. In the first case, the distances between the centers of mass of the two rings are $5.97 \AA$ and $5.66 \AA$, while in the second they are $4.89 \AA$ and $4.79 \AA$, for SAS and VDW structures respectively. This indicates that edge-to-face interactions contribute predominantly to aggregation, also considering that the $\pi-\pi$ stacking is sterically hindered. Moreover, it is interesting to observe that the calculated edge-to-face distance is 

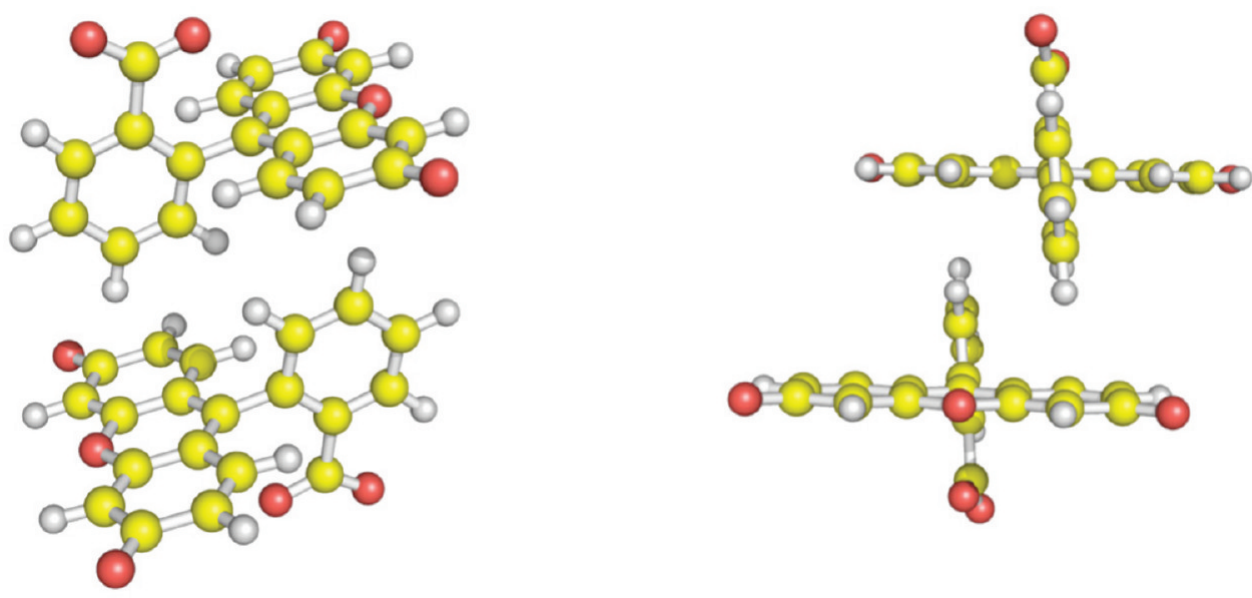

Figure 4. Sodium fluorescein dimer structure determined at the M062X/6-311+G(2d,2p) level using the SAS methodology to compute the solute-solvent boundary.
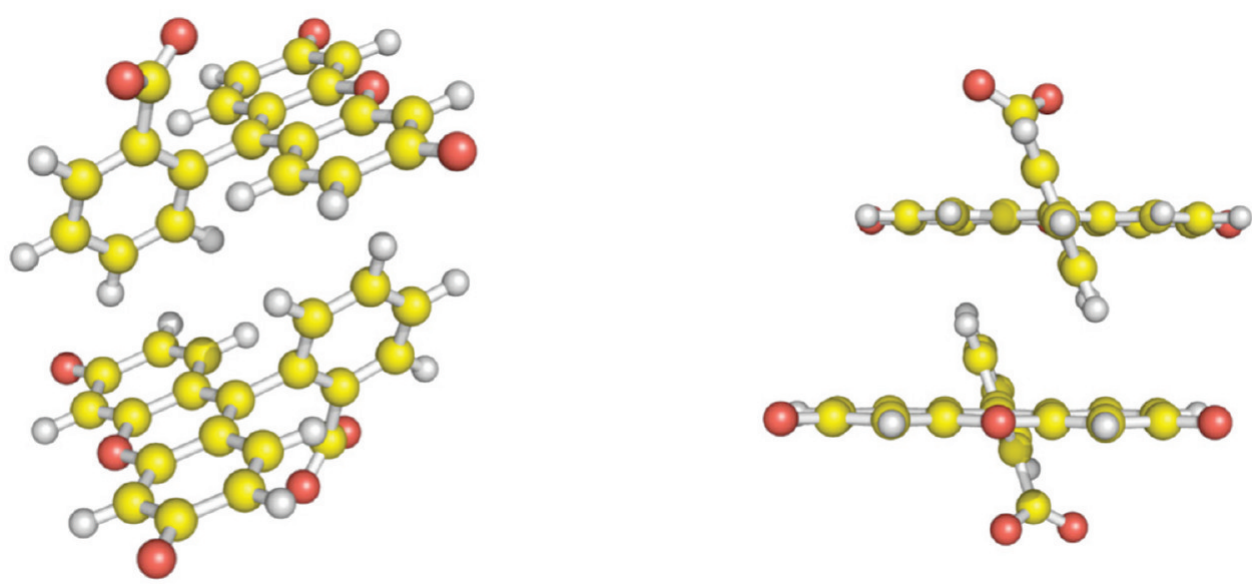

Figure 5. Sodium fluorescein dimer structure determined at the M062X/6-311+G(2d,2p) level using the VDW methodology to compute the solute-solvent boundary.

comparable with the $4.97 \AA$ calculated by Lee et al. at the MP2/ aug-cc-pVDZ level of theory for the benzene dimer. ${ }^{51}$

As mentioned, the interaction energy depends both from the functional used and from the structure. The interaction energies computed at different levels of theory using the structures optimized at the M062X/6-311+G(2d,2p) level using the SAS and VDW solute-solvent surfaces are reported in Table 1.

The analysis of the computational results shows that the B3LYP functional, which does not include the contribution of dispersion, predicts that the interaction is non binding. Also, the M05 density functional is not able to predict a dimer structure for calculations performed with the SAS structure. However, it must be pointed out that it is probably not the best choice for such system, since M05 performs better with structures involving transition metals. ${ }^{52}$ All of the remaining density functionals predict that both dimer structures are energetically stable. According to literature computational benchmarks for structures characterized by $\pi-\pi$ stacking, ${ }^{42}$ the most reliable functionals should be the M062X and M06HF density functionals. Also, Suresh et al. ${ }^{53}$ found that the MPWB $1 \mathrm{~K}^{54}$ density functional should be suitable to study systems involving $\pi-\pi$ stacking. It is thus interesting to observe that the interaction energies computed with these three functionals are in good agreement. Also, it is interesting to observe that both the SAS and VDW interaction energies calculated with the BLYP functional corrected for long-range interactions ${ }^{55}$ are in reasonable agreement with the predictions of the more sophisticated functionals, while the B97D seems to largely overestimate the VDW interaction energy. What strikes most however is the large difference between the interaction energies computed using the SAS and VDW solvent-solute surfaces. In judging the interaction energies reported in Table 1 , it should be considered that the experimental estimate of the interaction free energy of the fluorescein dimer is about $-1 \mathrm{kcal} / \mathrm{mol}$, thus significantly higher than the interaction energies computed using the VDW surface. Even if the interaction energies computed using the IEF-PCM solvation model are not rigorously free energies, as they do not include a gas phase entropic contribution due to vibration, rotation, and translation, at $300 \mathrm{~K}$ this is not likely to count for more than $2 \mathrm{kcal} / \mathrm{mol}$, which indicates that the VDW interaction energies are significantly larger than the experimental value. The decrease of interaction energies going from SAS to VDW surfaces is probably related to the balance between a binding component determined by dispersion interactions and a repulsive 
Table 1. Sodium Fluorescein Dimer Interaction Energies ( $\mathrm{kcal} / \mathrm{mol})$ Calculated on Structures Optimized at the M062X/6-311+G(2d,2p) Level Using the SAS and VDW Methodologies to Compute the Solvent-Solute Surfaces and Several Density Functionals

\begin{tabular}{lrc} 
density functional & interaction energy (SAS) & interaction energy (VDW) \\
M05 & 1.84 & -2.58 \\
M052X & -1.44 & -3.86 \\
M06 & -1.15 & -4.41 \\
M062X & -2.86 & -6.47 \\
M06L & -0.72 & -5.34 \\
M06HF & -2.92 & -8.62 \\
MPWB1K & -2.94 & -6.88 \\
MPW1B95 & -0.79 & -5.08 \\
B97D & -3.42 & -11.32 \\
LC-BLYP & -3.56 & -6.91 \\
B3LYP & 6.33 & 3.03 \\
\hline
\end{tabular}

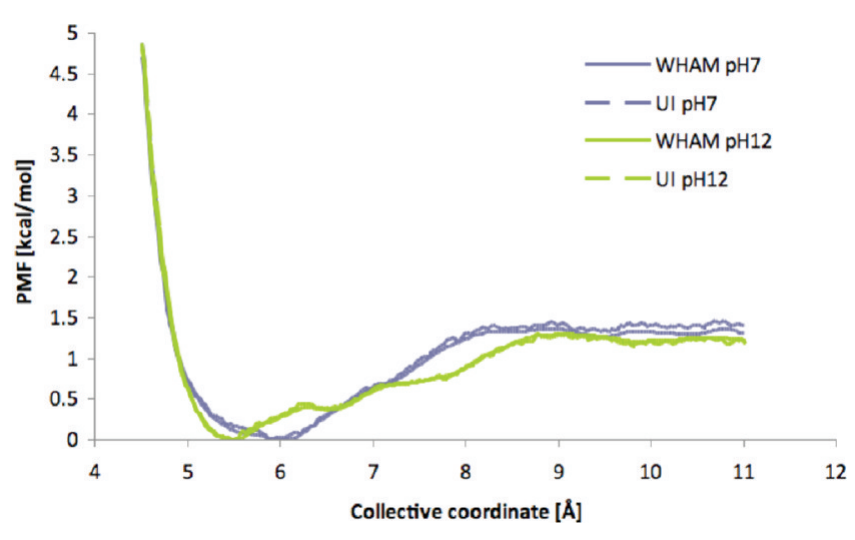

Figure 6. Potential of mean force between two fluorescein monomers calculated as a function of the distance between the centers of mass at two different $\mathrm{pHs}$.

component determined by the double negative charge of the anions, which gets screened when the solute-solvent distance decreases, as is the case for the VDW surface with respect to the SAS surface. Although at a first sight, it might be easy to conclude that the SAS methodology performs better than VDW for the system under investigation, it must however be considered that minimum energy structures such as those shown in Figures 4 and 5 are rarely reached in solution, due to thermal oscillations and interactions with the solvent. A similar effect of overestimation of interaction energy computed using ab initio methods with respect to that determined experimentally was recently observed by us when studying guanidiniocarbonylpyrrole host-guest complexes. ${ }^{56}$ In that case, $\mathrm{MD}$ computations were found to better perform in the calculation of the free interaction energy of the complexes, as they take into account more properly the intramolecular dynamics. To test whether a similar situation occurs also for fluorescein dimers MD was here used to determine free interaction energies for these molecules. The result of the simulations is described in the next section.

MD Analysis of the Sodium Fluorescein Dimer. The free energy of interaction of the fluorescein dimer was calculated using umbrella sampling, postprocessing the MD trajectories using two different approaches, WHAM and Umbrella

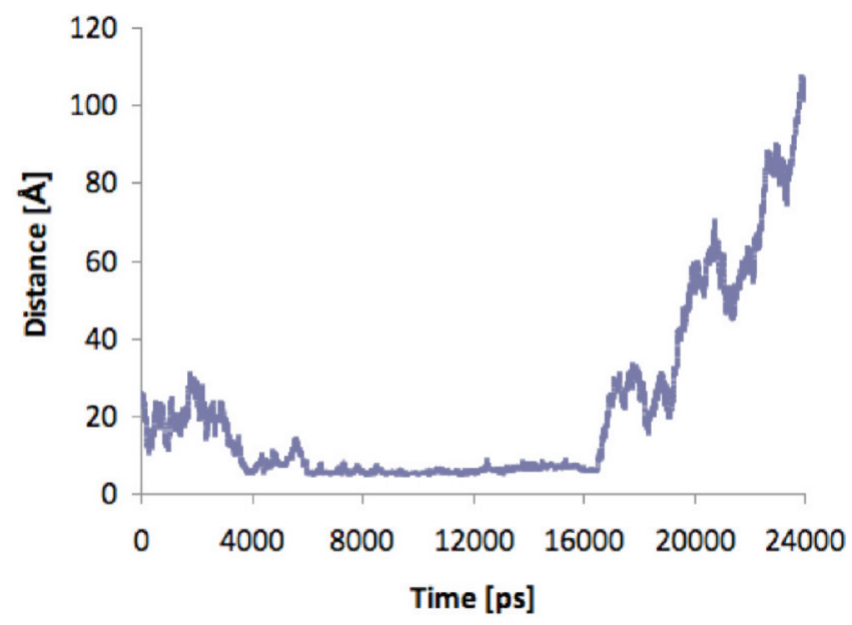

Figure 7. Evolution of the distance between the centers of mass of two fluorescein molecules that aggregate as a function of simulation time.

Integration, implemented as described by Salvalaglio et al. ${ }^{57}$ The collective coordinate adopted in the umbrella sampling simulations was defined as the distance between the centers of mass of each molecule. The potential of mean force so calculated is reported in Figure 6.

The interaction energy was calculated both at $\mathrm{pH} 7$ and 12, since though our main interest is toward the interaction energy in proximity of physiological conditions, the experimental value of the interaction energy was measured at a $\mathrm{pH}$ of $12 .{ }^{6,18}$ Interestingly, it was found that the $\mathrm{pH}$ does not influence significantly the free interaction energy of the dimer, while it has an impact on the minimum energy distance value. It was thus found that at a $\mathrm{pH}$ of 7 the interaction energy is $-1.33 \mathrm{kcal} \mathrm{mol}^{-1}$ and the profile minimum is at $5.89 \AA$, while at a $\mathrm{pH}$ of 12 the interaction energy rises slightly up to $-1.22 \mathrm{kcal} \mathrm{mol}^{-1}$, while the minimum is located at $5.5 \AA$. It is interesting to observe that the computed interaction energy is in good agreement with the experimental value of $-1 \mathrm{kcal} \mathrm{mol}^{-1}$., 18

To deepen the understanding of the system dynamics, the time evolution of the distances between the centers of mass of the molecules and of the rings involved in $\pi-\pi$ interactions was investigated. In order to study the properties of an unbiased system, the trajectories used in this analysis were not taken from the umbrella sampling simulations, but from the simulations used to compute the diffusion coefficient. As mentioned above, the analysis of the trajectories of this simulation had in fact evidenced that several dimers spontaneously form and decompose in the time span of the MD simulations. The onset of formation and decomposition of the complexes can be easily detected monitoring the time evolution of the distance between the centers of mass of the involved molecules. A typical output of this analysis is shown in Figure 7. As it can be observed, the formation of the aggregate takes place about $4 \mathrm{~ns}$ after the beginning of the simulation, after which the center of mass distance remains fixed for a time interval of about $10 \mathrm{~ns}$, and it is followed by the decomposition of the complex. In particular, 7 aggregates are formed in the $24 \mathrm{~ns}$ of the simulation, thus involving about $50 \%$ of the fluorescein molecules considered in the simulations. The average lifetime of the aggregates, shown in Figure 8, is about $5 \mathrm{~ns}$, while the average distance between the centers of mass is $6.07 \AA$, which is in good agreement with the PMF minimum calculated at a $\mathrm{pH}$ of 7 and with the $6.16 \AA$ value calculated using 

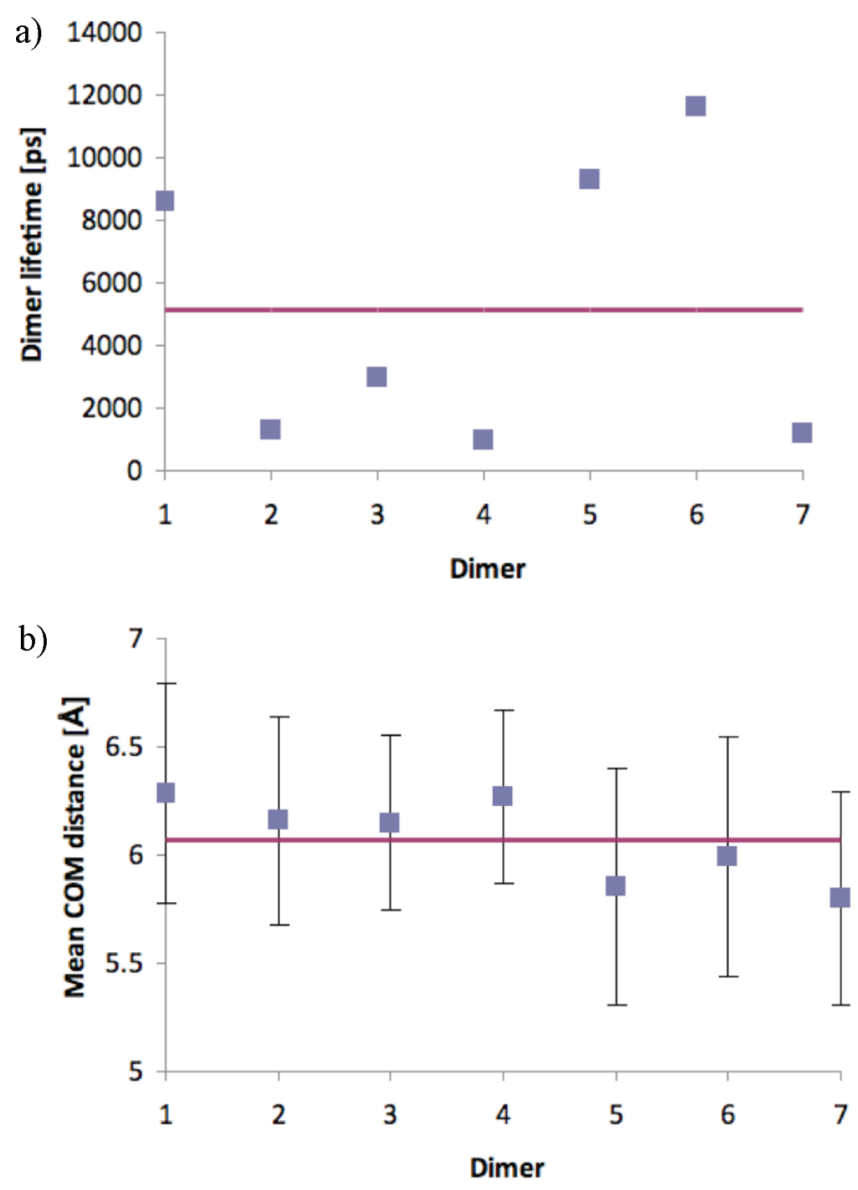

Figure 8. Average and specific lifetimes of the 7 dimers whose formation was observed in the simulation time span (a) and mean distances between the centers of mass (b).

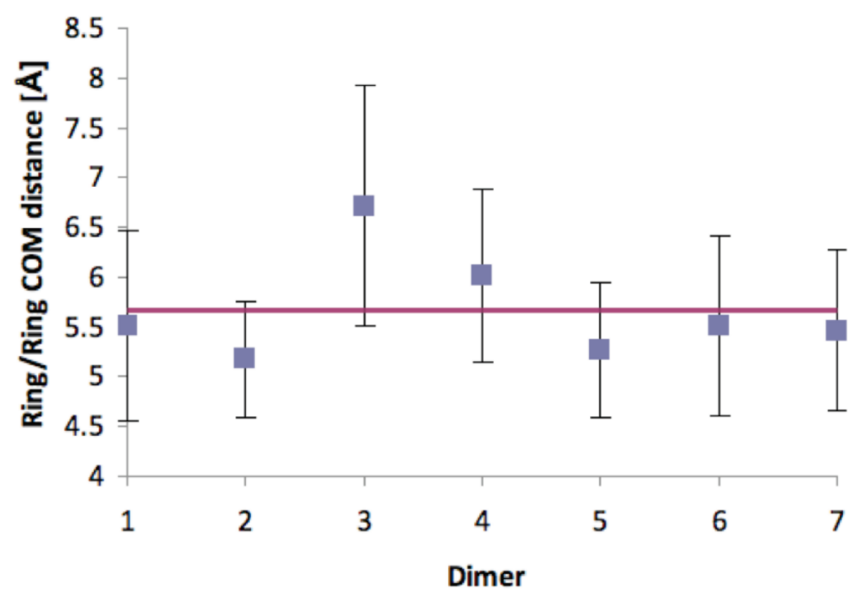

Figure 9. Mean distance between the centers of mass of the benzoic rings calculated in the sodium fluorescein complexes.

DFT and the VDW surface, while the distance calculated using the SAS surface, $6.64 \AA$, is significantly larger.

The same procedure can be used to investigate the evolution of other geometrical parameters of interest. In order to further characterize the dimer structure, the distances between the benzoic ring and that between the centers of mass of the rings

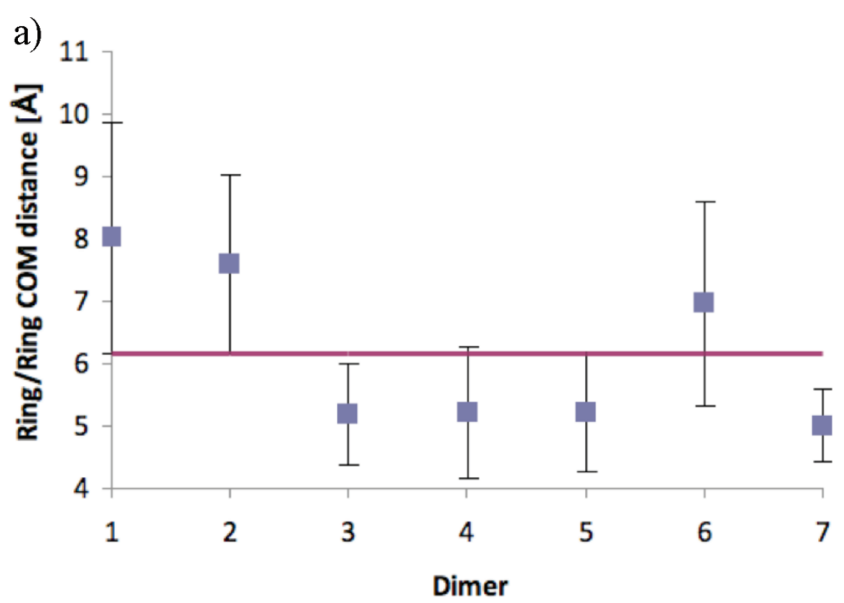

b)

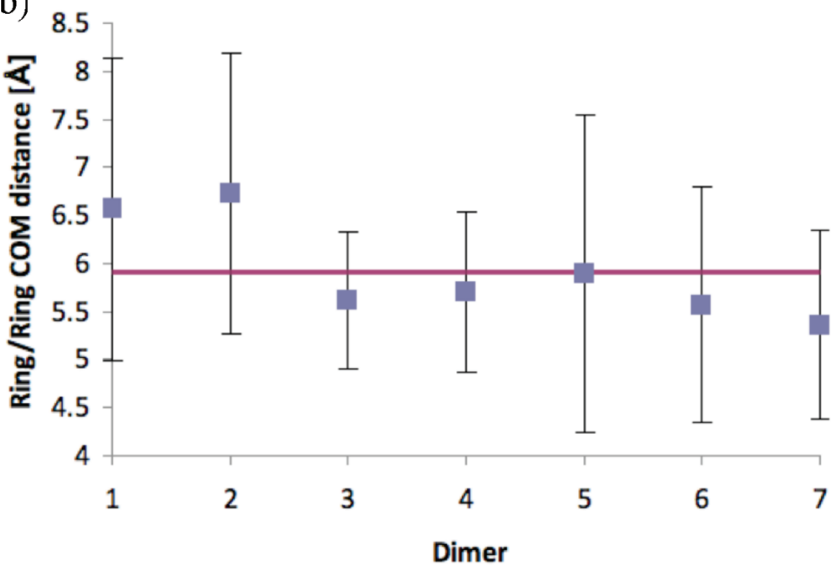

Figure 10. Mean centers of mass distances between the benzoic and phenoxy rings involved in edge to face $\pi-\pi$ interactions. The (a) and (b) plots correspond to the two distinct bonds that can be formed for each molecule.

involved in the $\pi-\pi$ edge to face interaction were monitored. The calculated parameters are reported in Figures 9 and 10.

According to the MD simulation results, the mean distance between the benzoic rings is $5.67 \AA$, which is in reasonable agreement with the $5.66 \AA$ determined using density functional theory and the VDW surface. Also in this case, the SAS DFT value, $5.97 \AA$, is larger than that calculated for the MD simulations. A significant difference between MD and DFT values can be observed for what concerns the rings involved in the edge to face $\pi-\pi$ interactions. Since the MD structures are not symmetric, the two edge to face $\pi-\pi$ bonds were considered as distinct and their mean distance was thus calculated separately. The average mean distances so determined are $6.17 \AA$ and $5.92 \AA$, thus significantly larger than the $4.89 \AA$ and $4.79 \AA$ determined using the SAS and VDW surfaces, respectively. These results clearly indicate that the symmetric minimum energy structure calculated using DFT is rarely reached during the MD simulations and that the dimer complexes oscillate between several possible structures, some of which are shown in Figure 11, all characterized by the establishment of van der Waals interactions and by the minimization of electrostatic repulsion between the negatively charged atoms. A structure of the trimer, which is formed for the very short timespan of 800 ps, is reported in Figure 12. Although the ff03 force field is probably unsuited to describe properly edge to face $\pi-\pi$ interactions, it can however 

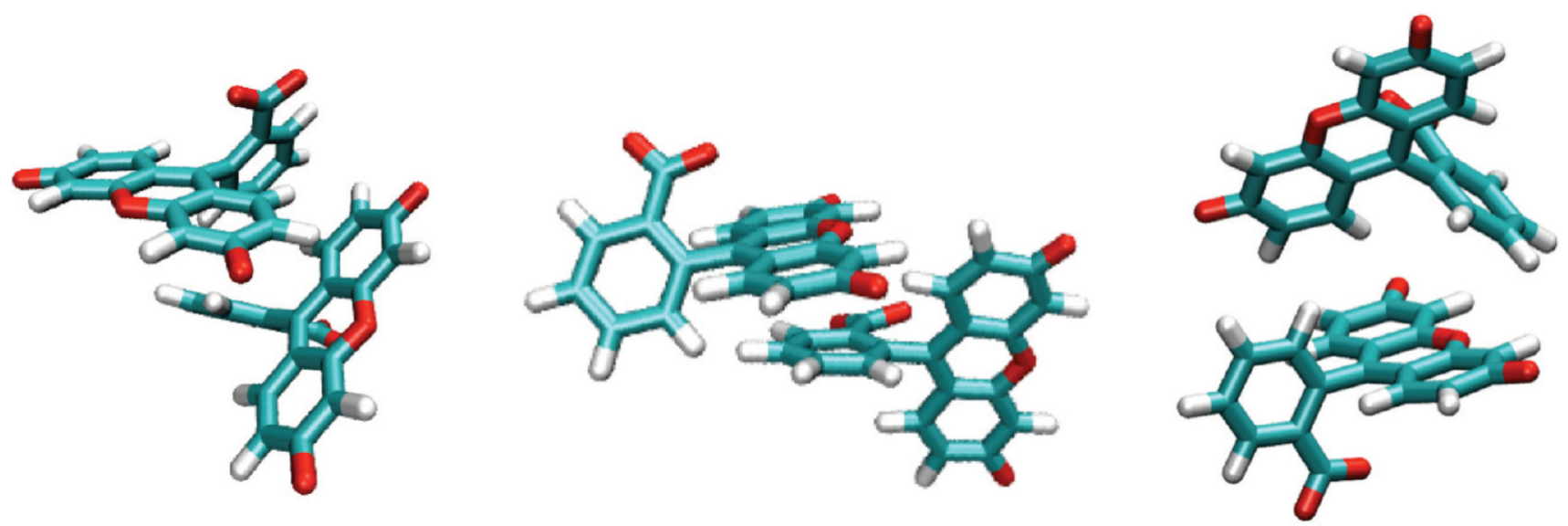

Figure 11. Examples of dimer structures observed during the MD simulation.

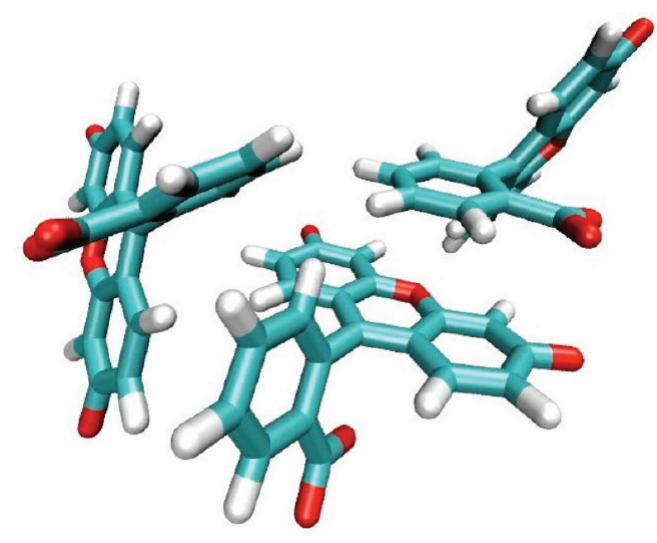

Figure 12. Example of trimer structure observed during the $\mathrm{MD}$ simulation.

be observed that it is able to predict a free interaction energy that is in good agreement with experimental data. It is here difficult to discriminate whether the dimer structure formed in solution by fluorescein is best described by the DFT model or by the highly mobile model predicted by MD simulations. The most reasonable conclusion is that both models represent a characteristic feature of this bond: energetic stability given by edge to face $\pi-\pi$ interactions and high mobility, and thus entropy, determined by the feeble interaction energy.

\section{CONCLUSIONS}

The subject of this work is the study of the dynamic behavior of sodium fluorescein in water through molecular dynamics and density functional theory calculations. The results of the MD simulations indicate that the $\mathrm{ff} 03$ force field, together with the fluorescein library developed in this work and reported as Supporting Information, can give an accurate description both of the diffusion of the fluorescein dianion in water and of its tendency to aggregate to form dimers. The calculated diffusion coefficient, $0.42 \times 10^{-5} \mathrm{~cm}^{2} \mathrm{~s}^{-1}$, is in fact in good agreement with the experimental values. It can thus be concluded that the methodology here developed can be extended to the study of fluorescein diffusion in gels.

The analysis of the MD trajectories revealed that in the concentration range at which fluorescein is usually loaded in gels there is a spontaneous and significant dimerization. In order to achieve a better understanding of this aspect, DFT simulations were performed to determine the minimum energy dimer structure and interaction energy. It was found that fluorescein aggregates in water in a symmetric structure that is stabilized by $\pi-\pi$ edge-to-face interactions. The dimer interaction energy was computed both through DFT and MD calculations. While the DFT calculations seem to significantly overestimate the interaction energy, a good agreement with experimental data was obtained from MD simulations, thus indicating that an approach able to account for the system dynamics is necessary when determining the interaction energy of this loosely bound complex. The calculated MD dimerization free energy is -1.3 $\mathrm{kcal} \mathrm{mol}^{-1}$, in good agreement with the experimental estimate of $-1 \mathrm{kcal} \mathrm{mol}^{-1}$.

\section{ASSOCIATED CONTENT}

S Supporting Information. Force field parameters and structures of DFT stationary points. This material is available free of charge via the Internet at http://pubs.acs.org.

\section{AUTHOR INFORMATION}

\section{Corresponding Author}

*E-mail: carlo.cavallotti@polimi.it.

\section{REFERENCES}

(1) Perale, G.; Arosio, P.; Moscatelli, D.; Barri, V.; Muller, M.; Maccagnan, S.; Masi, M. J. Controlled Release 2009, 136, 196.

(2) Santoro, M.; Marchetti, P.; Rossi, F.; Perale, G.; Castiglione, F.; Mele, A.; Masi, M. J. Phys. Chem. B 2011, 115, 2503.

(3) Perale, G.; Rossi, F.; Santoro, M.; Marchetti, P.; Mele, A.; Castiglione, F.; Raffa, E.; Masi, M. J. Biomed. Nanotechnol. 2011, 7, 1.

(4) Perale, G.; Veglianese, P.; Rossi, F.; Peviani, M.; Santoro, M.; Llupi, D.; Micotti, E.; Forloni, G.; Masi, M. Mater. Lett. 2011, 65, 1688.

(5) Rossi, F.; Perale, G.; Masi, M. Chem. Pap. 2010, 64, 573.

(6) Lòpez Arbeloa, I. J. Chem. Soc., Faraday Trans. 1981, 77, 1725.

(7) Lòpez Arbeloa, I. J. Chem. Soc., Faraday Trans. 1981, 77, 1735.

(8) Leonhardt, H.; Gordon, L.; Livingston, R. J. Phys. Chem. 1971, 76, 245.

(9) Sjoback, R.; Nygren, J.; Kubista, M. Spectrochim. Acta, Part A 1995, 51, L7.

(10) Krol, M.; Wrona, M.; Page, C. S.; Bates, P. A. J. Chem. Theory Comput. 2006, 2, 1520. 
(11) Jang, Y. H.; Hwang, S.; Chung, D. S. Abstr. Pap. Am. Chem. Soc. 2003, 225, U772.

(12) Jang, Y. H.; Hwang, S. G.; Chung, D. S. Chem. Lett. 2001, 1316.

(13) Batistela, V. R.; Cedran, J. C.; de Oliveira, H. P. M.; Scarminio,

I. S.; Ueno, L. T.; Machado, A. E. D.; Hioka, N. Dyes Pigm. 2010, 86, 15.

(14) Saltzman, W. M.; Radomsky, M. L.; Whaley, K. J.; Cone, R. A. Biophys. J. 1994, 66, 508.

(15) Radomsky, M. L.; Whaley, K. J.; Cone, R. A.; Saltzman, W. M. Biomaterials 1990, 11, 619.

(16) Soeller, C.; Jacobs, M. D.; Donaldson, P. J.; Cannell, M. B. J. Biomed. Opt. 2003, 8, 418.

(17) Valdes-Aguilera, O.; Neckers, D. C. Acc. Chem. Res. 1989, 22,171 .

(18) Rohatgi, K. K.; Singhal, G. S. J. Chem. Phys. 1966, 70, 1695.

(19) Lòpez Arbeloa, I. Dyes Pigm. 1983, 4, 213.

(20) Lòpez Arbeloa, I. Thermochim. Acta 1983, 60, 219.

(21) Lòpez Arbeloa, I. J. Photochem. 1982, 18, 161.

(22) Rohatgi, K. K.; Mukhopahyay, A. K. J. Phys. Chem. 1972, 76, 3970.

(23) De, S.; Das, S.; Girigoswami, A. Spectrochim. Acta, Part A 2005, $61,1821$.

(24) Das, S.; Chattopadhyay, A. P.; De, S. J. Photochem. Photobiol., A 2008, 197, 402.

(25) Daré-Doyen, S.; Doizi, D.; Guilbaud, P.; Djedaini-Pilard, F.; Perly, B.; Millié, P. J. Phys. Chem. B 2003, 107, 13803.

(26) Chuichay, P.; Vladimirov, E.; Siriwong, K.; Hannongbua, S.; Rosch, N. J. Mol. Model. 2006, 12, 885.

(27) Ivanova, A.; Jezierski, G.; Vladimirov, E.; Rosch, N. Biomacromolecules 2007, 8, 3429.

(28) Ilich, P.; Mishra, P. K.; Macura, S.; Burghardt, T. P. Spectrochim. Acta, Part A 1996, 52, 1323.

(29) Becke, A. D. J. Chem. Phys. 1993, 98, 5648.

(30) Lee, C.; Yang, W.; Parr, R. G. Phys. Rev. B 1988, 37, 785.

(31) Frisch, M. J.; Trucks, G. W.; Schlegel, H. B.; Scuseria, G. E.; Robb, M. A.; Cheeseman, J. R.; Scalmani, G.; Barone, V.; Mennucci, B.; Petersson, G. A.; Nakatsuji, H.; Caricato, M.; Li, X.; Hratchian, H. P.; Izmaylov, A. F.; Bloino, J.; Zheng, G.; Sonnenberg, J. L.; Hada, M.; Ehara, M.; Toyota, K.; Fukuda, R.; Hasegawa, J.; Ishida, M.; Nakajima, T.; Honda, Y.; Kitao, O.; Nakai, H.; Vreven, T.; Montgomery, J., J., A.; Peralta, J. E.; Ogliaro, F.; Bearpark, M.; Heyd, J. J.; Brothers, E.; Kudin, K. N.; Staroverov, V. N.; Kobayashi, R.; Normand, J.; Raghavachari, K.; Rendell, A.; Burant, J. C.; Iyengar, S. S.; Tomasi, J.; Cossi, M.; Rega, N.; Millam, N. J.; Klene, M.; Knox, J. E.; Cross, J. B.; Bakken, V.; Adamo, C.; Jaramillo, J.; Gomperts, R.; Stratmann, R. E.; Yazyev, O.; Austin, A. J.; Cammi, R.; Pomelli, C.; Ochterski, J. W.; Martin, R. L.; Morokuma, K.; Zakrzewski, V. G.; Voth, G. A.; Salvador, P.; Dannenberg, J. J.; Dapprich, S.; Daniels, A. D.; Farkas, Ö.; Foresman, J. B.; Ortiz, J. V.; Cioslowski, J.; Fox, D. J. Gaussian 09, Revision A.1; Gaussian, Inc.: Wallingford CT, 2009.

(32) Ditchfield, R.; Hehre, W. J.; Pople, J. A. J. Chem. Phys. 1971, 54, 724.

(33) Mennucci, B.; Cances, E.; Tomasi, J. J. Phys. Chem. B 1997, 101, 10506.

(34) Duan, Y.; Wu, C.; Chowdhury, S.; Lee, M. C.; Xiong, G. M.; Zhang, W.; Yang, R.; Cieplak, P.; Luo, R.; Lee, T.; Caldwell, J.; Wang, J. M.; Kollman, P. J. Comput. Chem. 2003, 24, 1999.

(35) Clark, T.; Chandrasekhar, J.; Spitznagel, G. W.; Schleyer, P. v. R. J. Comput. Chem. 1983, 4, 294.

(36) Bayly, C. I.; Cieplak, P.; Cornell, W. D.; Kollman, P. A. J. Phys. Chem. 1993, 97, 10269.

(37) Cornell, W. D.; Cieplak, P.; Bayly, C. I.; Kollman, P. A. J. Am. Chem. Soc. 1993, 115, 9620.

(38) Case, D. A.; Darden, T. A.; Cheatham, I., T.E. ; Simmerling, C. L.; Wang, J.; Duke, R. E.; Luo, R; Crowley, M.; Walker, R C.; Zhang, W.; Merz, K. M.; Wang, B.; Hayik, S.; Roitberg, A.; Seabra, G.; Kolossváry, I.; Wong, K. F.; Paesani, F.; Vanicek, J.; Wu, X.; Brozell, S. R.; Steinbrecher, T.; Gohlke, H.; Yang, L.; Tan, C.; Mongan, J.; Hornak, V.; Cui, G.; Mathews, D. H.; Seetin, M. G.; Sagui, C.; Babin, V.; Kollman, P. A. Amber 10; University of California: San Francisco, 2008.
(39) Jorgensen, W. L.; Chandrasekhar, J.; Madura, J. D.; Impey, R. W.; Klein, M. L. J. Chem. Phys. 1983, 79, 926.

(40) Ryckaert, J. P.; Ciccotti, G.; Berendsen, H. J. Comput. Phys. 1977, 23, 327.

(41) Frenkel, D.; Smit, B. Understanding Molecular Simulation: From Algorithms to Applications, 2nd ed.; Academic Press: San Diego, 2002.

(42) Zhao, Y.; Truhlar, D. G. Theor. Chem. Acc. 2008, 120, 215.

(43) Zhao, Y.; Truhlar, D. G. Chem. Phys. Lett. 2011, 502, 1.

(44) Gu, J.; Wang, J.; Leszczynski, J.; Xie, Y.; Schaefer, H. F., III Chem. Phys. Lett. 2008, 459, 164.

(45) Frisch, M. J.; Pople, J. A.; Binkley, J. S. J. Chem. Phys. 1984, $80,3265$.

(46) Tomasi, J.; Mennucci, B.; Cammi, R. Chem. Rev. 2005, 105, 2999.

(47) Roux, B. Comput. Phys. Commun. 1995, 91, 275.

(48) Kumar, S.; Bouzida, D.; Swendsen, R. H.; Kollman, P. A.; Rosenberg, J. M. J. Comput. Chem. 1992, 13, 1011.

(49) Kastner, J.; Thiel, W. J. Chem. Phys. 2005, 123.

(50) Kobrak, M. N. J. Comput. Chem. 2003, 24, 1437.

(51) Lee, E. C.; Kim, D.; Jurecka, P.; Tarakeshwar, P.; Hobza, P.; Kim, K. S. J. Phys. Chem. A 2007, 111, 3446.

(52) Zhao, Y.; Schultz, N. E.; Truhlar, D. G. J. Chem. Theory Comput. 2006, 2, 364.

(53) Suresh, C. H.; Mohan, N.; Vijayalakshmi, K. P.; George, R.; Mathew, J. M. J. Comput. Chem. 2009, 30, 1392.

(54) Zhao, Y.; Truhlar, D. G. J. Phys. Chem. A 2004, 108, 6908.

(55) Iikura, H.; Tsuneda, T.; Yanai, T.; Hirao, K. J. Chem. Phys. 2001, 115,3540

(56) Moiani, D.; Cavallotti, C.; Famulari, A.; Schmuck, C. Chem.-Eur. J. 2008, 14, 5207.

(57) Salvalaglio, M.; Muscionico, I.; Cavallotti, C. J. Phys. Chem. B 2010, 114, 14860 . 